\title{
Supernormal scotopic ERG in cone dystrophy
}

\author{
KENNETH R. ALEXANDER AND GERALD A. FISHMAN \\ From the Department of Ophthalmology, University of Illinois Eye and Ear Infirmary, Chicago, USA
}

SUMMARY Three patients with a bull's-eye macular lesion and other signs characteristic of cone dystrophy gave an unusual ERG finding. In response to a white flash of moderate intensity the scotopic b-wave amplitude was considerably larger than normal. One patient had elevated rod thresholds and nyctalopia, while the other 2 had normal rod sensitivity associated with the supernormal scotopic b-wave amplitude. In the latter 2 patients the abnormal ERG pattern was unchanged for 4 years and 7 years respectively. This atypical finding, of a supernormal scotopic b-wave amplitude in response to light of moderate intensity, appears to characterise a subgroup of patients with cone dystrophy, probably of autosomal recessive inheritance. The pathogenesis of the abnormal ERG remains uncertain.

Cone dystrophy is a group of disorders often characterised by an atrophic macular lesion, typically bull's-eye-like in appearance, associated with reduced visual acuity, poor colour vision, light sensitivity, and in some instances nystagmus. ${ }^{1-6}$ The dystrophy may be diffuse, with a reduced or nondetectable photopic ERG, or localised in the macula, with a normal photopic electroretinogram (ERG). ${ }^{4}$ Abnormalities of rod function are sometimes also apparent, including a scotopic ERG which is reduced in amplitude (cone-rod dystrophy). ${ }^{7 \rightarrow}$ Recently an unusual type of cone dystrophy has been reported in which the rod ERG is subnormal at low flash intensities but is supernormal with brighter flashes. ${ }^{10}$ In the 2 patients described the cone dystrophy occurred in association with nyctalopia.

The purpose of this report is to describe 3 cases of a supernormal scotopic ERG associated with an atrophic macular lesion. One case is similar to those of cone dystrophy with nyctalopia reported by Gouras et al. ${ }^{10}$ The other 2 cases seem to represent a previously unreported clinical entity of cone dystrophy with a supernormal scotopic ERG without nyctalopia. The latter patients demonstrate that a supernormal scotopic ERG can occur without impaired rod function.

\section{Materials and methods}

The procedures for recording the ERG have been described elsewhere. ${ }^{11}$ Briefly, the patients' pupils Correspondence to Dr Gerald A. Fishman, University of Illinois Eye and Ear Infirmary, 1855 W. Taylor Street, Chicago, IL 60612, USA. were dilated with $10 \%$ phenylephrine and $1 \%$ cyclopentolate drops, following the application of $5 \%$ proparacaine hydrochloride as a topical anaesthetic. The ERGs were recorded with a Burian-Allen monopolar electrode wetted with methylcellulose. Responses were elicited by a Grass PS-22 photostimulator that illuminated a diffusing sphere to provide Ganzfeld stimulation. Signals were amplified by a Grass preamplifier and by a Tektronix dual trace amplifier. Results were displayed on an oscilloscope and photographed with an oscilloscope camera.

Subjects were adapted for 5 minutes to a Ganzfeld illumination of $1.7 \mathrm{log} \mathrm{cd} \mathrm{m}^{-2}$, and then a single-flash photopic response was recorded against a background of $1.4 \log \mathrm{cd} \mathrm{m}^{-2}$, using the unattenuated I-16 setting, which had nominal intensity of $1.5 \times 10^{6}$ candle power and a duration at half-amplitude of $10 \mu$ s. The background was extinguished, and single-flash recordings were obtained after $5,10,15,20$, and 30 minutes of dark adaptation, again with the use of the unattenuated I-16 setting. A single-flash response to dim blue light (produced by Wratten 47 and $0.5 \mathrm{log}$ unit neutral density filters and an I-4 setting) was also obtained after 30 minutes of dark adaptation to stimulate the rod system preferentially. An amplitude-intensity function was then obtained. The I-16 flash intensity was attenuated by $3.5 \mathrm{log}$ units of neutral density filtering, and a single-flash white-light ERG response was recorded. The flash intensity was then systematically increased in $0.5 \log$ unit steps, and the ERG was recorded at each intensity. A brief period of dark adaptation of approximately 2 minutes' duration followed each flash presentation. 
Static profile perimetry was performed with a Tubinger perimeter. The patients' pupils were dilated as above, and then patients were dark-adapted for at least 40 minutes. Absolute thresholds were obtained across the visual field for a test stimulus (TS) that was $104^{\prime}$ in diameter, $500 \mathrm{~ms}$ in duration, and either of a long wavelength (Oculus glass filter, bandpass $>620$ $\mathrm{nm}$ ) or a middle wavelength (Oculus interference filter, $\lambda_{\max }=500 \mathrm{~nm}$ ). The relative threshold values for the 2 chromatic stimuli at each retinal location indicate whether rods or cones determine the absolute threshold. ${ }^{12}$ The sensitivity of the cone system was further assessed by light-adapted flicker perimetry. ${ }^{13}$ The threshold luminance for detecting flicker across the visual field was measured with a 25 $\mathrm{Hz}$ white $\mathrm{TS}, 104^{\prime}$ in diameter and 3 seconds in duration, presented against a Ganzfeld background of $0.5 \log \mathrm{cd} \mathrm{m}^{-2}$ to suppress the rod system.

\section{Case reports and results}

\section{CASE 1: CONE-ROD DYSTROPHY}

A 19-year-old black woman was referred for poor visual acuity of approximately 2 years' duration. The patient's only other complaint was that she seemed to take longer than usual to adjust to darkness. She gave no specific history of poor colour vision or photosensitivity. The patient had noted no further reduction in central acuity, or increased difficulty in adjusting to darkness over the past 2 years. The patient was in good health and had no known systemic disorders. She was taking Ortho-Novum (tablets containing norethisterone) for birth control. A review of the family pedigree indicated that the patient has a brother, age 26 , who also has reduced visual acuity that is uncorrectable with glasses. $\mathrm{He}$ was unavailable for examination. Two other brothers and two sisters were, by history, normal.

Vision was correctable to $20 / 100+1$ OD with a $-1 \cdot 75$ and $20 / 100+1$ OS with $a-3 \cdot 50$. The patient could read $\mathrm{J}-2$ at 6 inches without correction. External examination showed 20 prism dioptres of left exotropia for near and distance, but was otherwise normal. Slit-lamp examination of the cornea, anterior chamber, iris, and lens was similarly normal. The vitreous was moderately fibrillar but otherwise unremarkable. Fundus examination showed normal optic discs. The retinal arterioles were mildly attenuated. Both maculas showed bilateral atrophiclooking lesions that had a bull's-eye appearance (the right fundus is shown in Fig. 1). Examination of the peripheral retina showed no evidence of pigmentary abnormalities.

Visual field testing, with the 4-e-II and 2-e-II test targets on a Goldmann perimeter, showed no peripheral depression or central scotomas. Colour

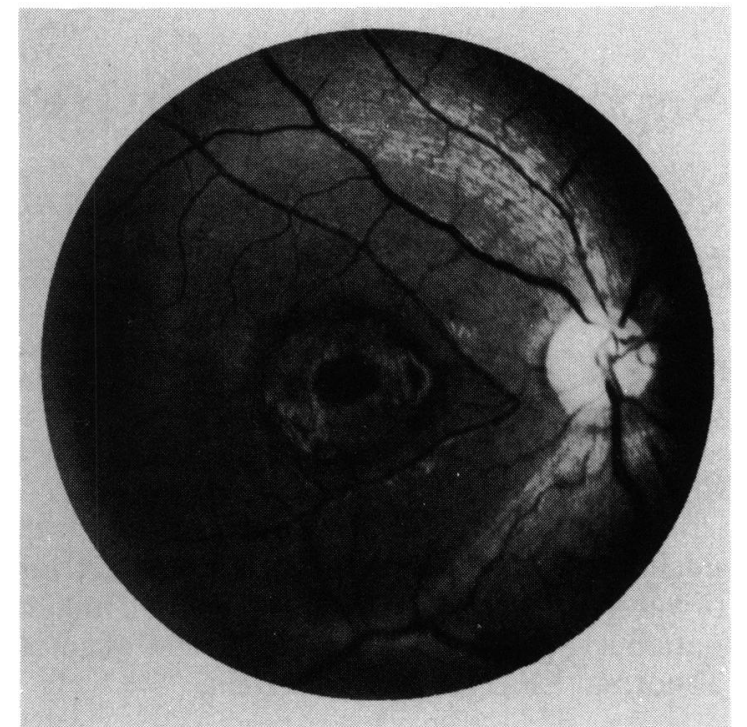

Fig. 1 Fundus photograph of the right eye of patient 1 , showing a bull's-eye-like macular lesion.

vision was abnormal, with a large increase in the number of errors on the FM 100-hue test (with no specific axis), and a shift toward the red setting on the Nagel anomaloscope.

Absolute thresholds across the horizontal meridian of the visual field are shown in Fig. 2. Data are plotted in photopic units; coincident data points for the middle and long wavelength test stimuli represent

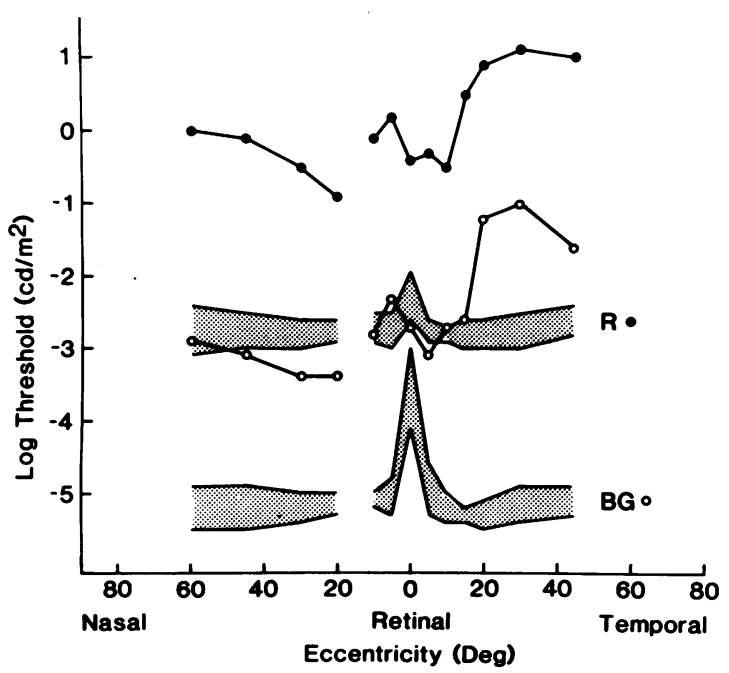

Fig. 2 Absolute thresholds across the horizontal meridian of the right eye of patient 1 for long wavelength (filled circles) and middle wavelength (open circles) test stimuli. Shaded regions represent the range of thresholds for 5 normal observers. 


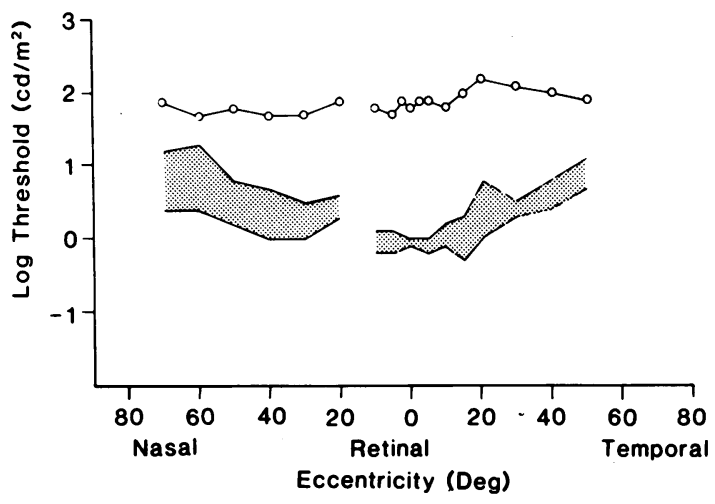

Fig. 3 Light-adapted flicker thresholds across the horizontal meridian of the right eye of patient 1 for a $25 \mathrm{~Hz}$ white test stimulus (open circles). Shaded region represents the range of flicker thresholds for four normal observers.

cone-mediated absolute thresholds, whereas a vertical separation of $2.6 \log$ units indicates rod-mediated thresholds for both test stimuli. Intermediate vertical separations indicate that cones detect the long wavelength TS, while rods detect the middle wavelength TS. ${ }^{12}$ Dotted regions in Fig. 2 represent the range of absolute threshold settings for 5 normal subjects. For this patient absolute thresholds are mediated by rods throughout the horizontal meridian. (The absence of a central region of cone-mediated thresholds is due to the patient's use of a nonfoveal region for fixation.) Rod thresholds are elevated by at least $2 \log$ units throughout the retina, supporting the patient's complaint of nyctalopia.

Cone sensitivity across the horizontal meridian of the visual field, as measured by light-adapted flicker perimetry, is shown in Fig. 3, with the dotted region representing the range of flicker thresholds for 4 normal observers. The patient had a loss of cone sensitivity at all tested locations, indicating the diffuse nature of the dystrophy.

The patient's ERG responses in comparison with those of a normal subject are shown in Fig. 4. Vertical bars on the normal tracings represent $90 \%$ confidence limits for the mean of 21 (photopic white flash), 18 (scotopic white flash), or 23 (blue flash) normal responses. The top tracing shows the light-adapted photopic ERG. The a-wave is normal in amplitude but prolonged in implicit time, while the b-wave amplitude is reduced to approximately $50 \%$ of normal, and the implicit time is prolonged (see Table 1). The next 3 tracings show the single-flash ERG response after 5,15 , and 30 minutes of dark adaptation. The a-wave amplitude and implicit time are

Table 1 Summary of ERG responses

\begin{tabular}{|c|c|c|c|c|c|c|}
\hline \multirow[t]{2}{*}{ Patient no. } & & \multicolumn{2}{|c|}{ a-wave } & \multicolumn{3}{|l|}{$b$-wave } \\
\hline & & Amplitude* & Implicit time ${ }^{\dagger}$ & Amplitude $\ddagger$ & \multicolumn{2}{|c|}{ Implicit time§ } \\
\hline $\begin{array}{l}1 \\
2 \\
3\end{array}$ & & $\begin{array}{l}\text { Normal } \\
\text { Normal } \\
\text { Normal }\end{array}$ & $\begin{array}{l}\text { Prolonged }(24 \mathrm{~ms}) \\
\text { Normal } \\
\text { Normal }\end{array}$ & $\begin{array}{l}\text { Reduced }(50 \mu \mathrm{V}) \\
\text { Normal } \\
\text { Normal }\end{array}$ & \multicolumn{2}{|c|}{$\begin{array}{l}\text { Prolonged (42 ms) } \\
\text { Normal } \\
\text { Normal }\end{array}$} \\
\hline \multicolumn{7}{|c|}{ Scotopic flash } \\
\hline \multirow[t]{3}{*}{ Patient no. } & \multicolumn{4}{|l|}{ White flash } & \multicolumn{2}{|l|}{ Blue flash } \\
\hline & \multicolumn{2}{|l|}{ a-wave } & \multicolumn{2}{|l|}{$b$-wave } & \multicolumn{2}{|l|}{$b$-wave } \\
\hline & Amplitude & & Amplitude** & Implicit time & Amplitude ${ }^{\dagger \dagger}$ & Implicit time $\ddagger \ddagger$ \\
\hline 1 & Normal & & $\begin{array}{l}\text { Supernormal } \\
(750 \mu \mathrm{V})\end{array}$ & Normal & $\begin{array}{l}\text { Reduced } \\
(80 \mu \mathrm{V})\end{array}$ & $\begin{array}{l}\text { Prolonged } \\
(108 \mathrm{~ms})\end{array}$ \\
\hline 2 & Normal & & $\begin{array}{l}\text { Supernormal } \\
(800 \mu \mathrm{V})\end{array}$ & Normal & $\begin{array}{l}\text { Supernormal } \\
(460 \mu V)\end{array}$ & Normal \\
\hline 3 & Normal & & $\begin{array}{l}\text { Supernormal } \\
(820 \mu \mathrm{V})\end{array}$ & Normal & Normal & Normal \\
\hline
\end{tabular}

*'Normal' indicates that the value was within $90 \%$ confidence limits for the mean.

$+90 \%$ confidence limits $=14$ to $20 \mathrm{~ms}, \mathrm{n}=30$.

$\$ 90 \%$ confidence limits $=100$ to $180 \mu \mathrm{V}, \mathrm{n}=21$.

$\$ 90 \%$ confidence limits $=26$ to $34 \mathrm{~ms}, \mathrm{n}=21$.

** $90 \%$ confidence limits $=400$ to $610 \mu \mathrm{V}, \mathrm{n}=18$.

$++90 \%$ confidence limits $=190$ to $300 \mu \mathrm{V}, \mathrm{n}=29$.

$\$ \ddagger 90 \%$ confidence limits $=80$ to $92 \mathrm{~ms}, \mathrm{n}=21$. 


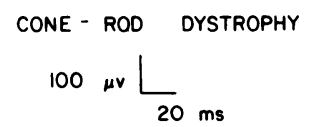

Fig. 4 ERG responses for the right eye of patient 1 (left column) and for a typical normal observer (right column) under the conditions indicated. Vertical bars represent $90 \%$ confidence limits for the mean of the normal responses. Calibration bars are shown at the top.
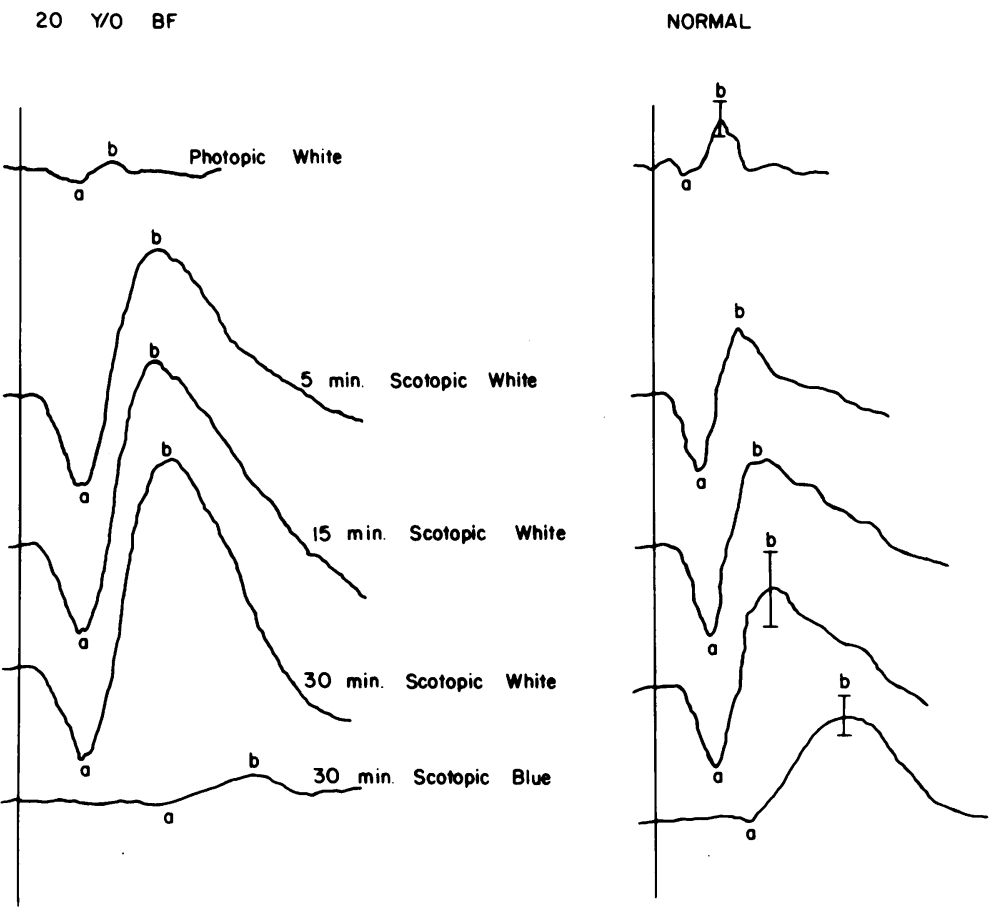

within normal limits, but the b-wave amplitude is clearly supernormal, although normal in implicit time (Table 1). The bottom tracing represents the ERG response to a dim blue flash after 30 minutes of dark adaptation. While the b-wave amplitude to a bright flash is supernormal, the b-wave response to the dim blue flash is subnormal in amplitude and prolonged in implicit time. The b-wave amplitude-intensity functions for this patient and for 2 normal subjects are shown in Fig. 5. At low flash intensities the bwave amplitude for the cone-rod dystrophy patient is subnormal, but the amplitude rapidly overtakes the normal response and becomes supernormal at high flash intensities.

CASE 2: CONE DYSTROPHY

A 30-year-old white woman first noted blurred vision in each eye at approximately age 23 . The blurred vision slowly increased in severity over the ensuing 3 years. When first seen by the authors at age 26 she gave no history of photosensitivity, poor colour vision, or nyctalopia. The patient was otherwise in good health and was not taking any medication. A review of the family history was pertinent in that her maternal grandparents were first cousins. There were no other known family members with ocular disease.

When the patient was initially seen, visual acuity was correctable to $20 / 40+2$ OD with a +1.00 and 20/40-2 OS with a $+1 \cdot 00$. External examination showed no abnormalities of ocular motility. Slit-lamp examination of the cornea, anterior chamber, lens, and vitreous were normal. Fundus examination disclosed normal optic discs and retinal vessels. There was no evidence of any peripheral pigmentary abnormalities. However, the patient had bilateral bull's-eye macular lesions (Fig. 6). The bull's-eye appearance was accentuated on fluorescein angiography (Fig. 7).

The patient was seen subsequently at age 30,4 years after her initial examination. Visual acuity had decreased to 20/200 OD but was still 20/40-2 OS. Grossly, the bull's-eye lesions were somewhat more extensive than on her initial examination. The optic discs, retinal vessels, and peripheral retina remained normal. At this time visual field testing with the 2-e-II target on a Goldmann perimeter showed normal 


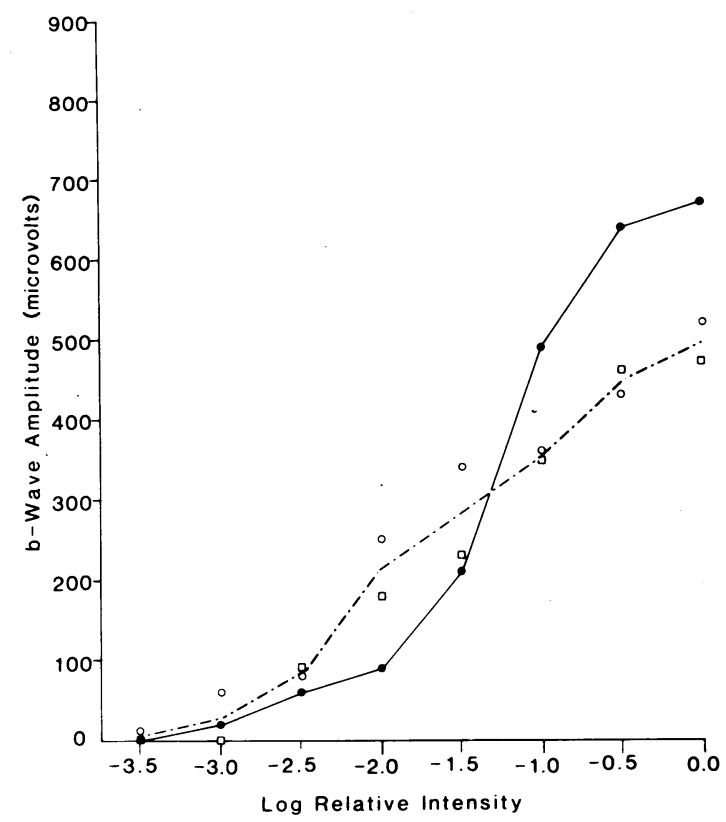

Fig. $5 A$-wave amplitude-intensity function for the left eye of patient 1 (filled circles) and for 2 normal observers (open symbols). Dashed line connects the mean of the 2 normal values.

peripheral boundaries but bilateral central scotomas. Colour vision testing with the FM 100-hue and D-15 tests showed normal findings. On the Nagel anomaloscope the patient showed a shift toward red in the Rayleigh equation.

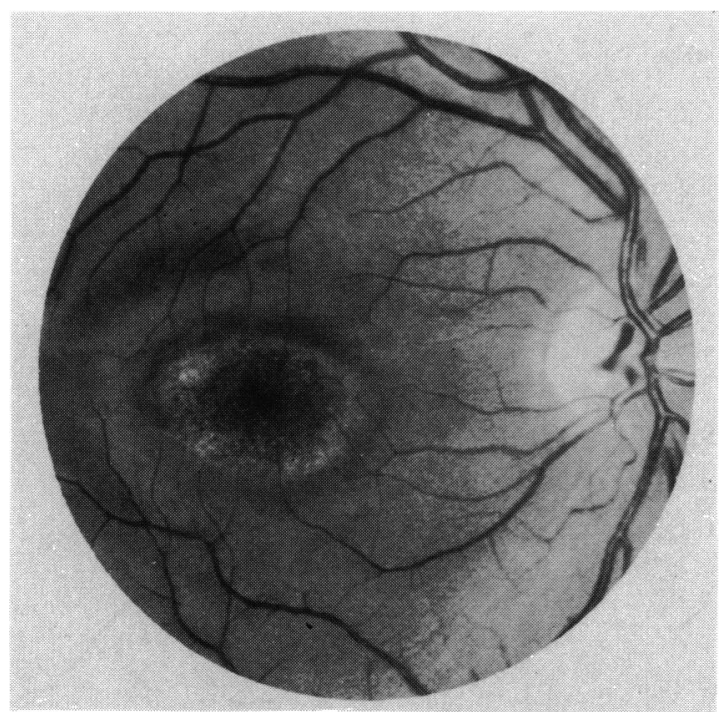

Fig. 6 Fundus photograph of the right eye of patient 2, showing a bull's-eye-like macular lesion.

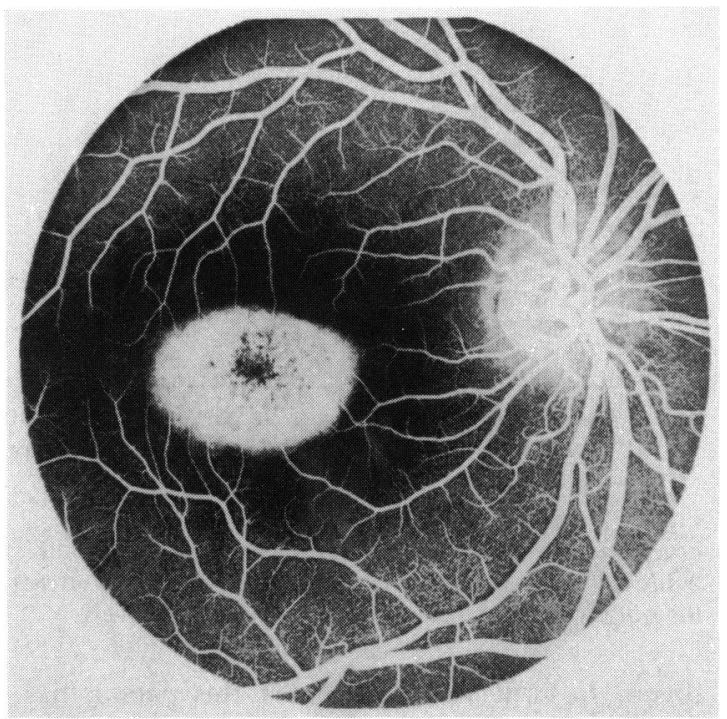

Fig. 7 Fluorescein angiogram of the right eye of patient 2, in which a hyperfluorescent window defect accentuates the bull's-eye macular lesion shown in Fig. 6.

Absolute thresholds across the horizontal meridian of the visual field are shown in Fig. 8. The foveal threshold for the long wavelength (cone-detected) TS is somewhat elevated. In the parafoveal and peripheral retina absolute thresholds are rodmediated and within normal limits. Cone thresholds obtained by flicker perimetry are shown in Fig. 9. In the fovea the flicker threshold is elevated, but cone flicker thresholds are within normal limits outside the

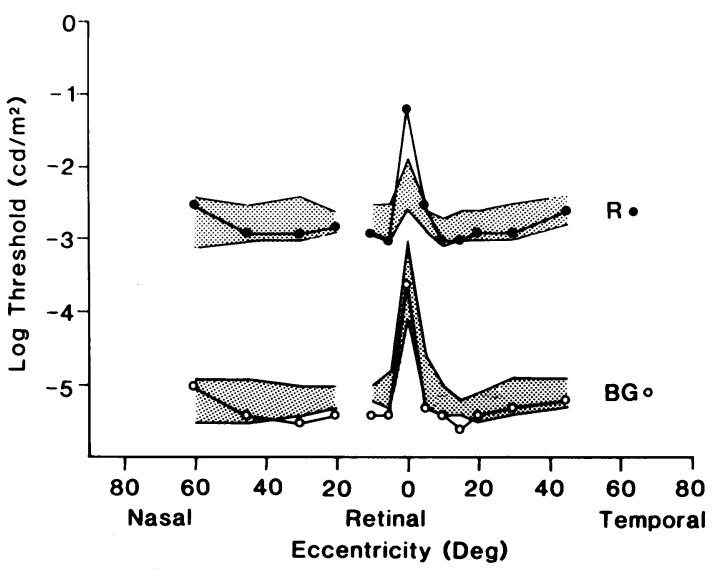

Fig. 8 Absolute thresholds across the horizontal meridian of the right eye of patient 2 for long wavelength (filled circles) and middle wavelength (open circles) test stimuli. Shaded regions represent the range of thresholds for 5 normal observers. 


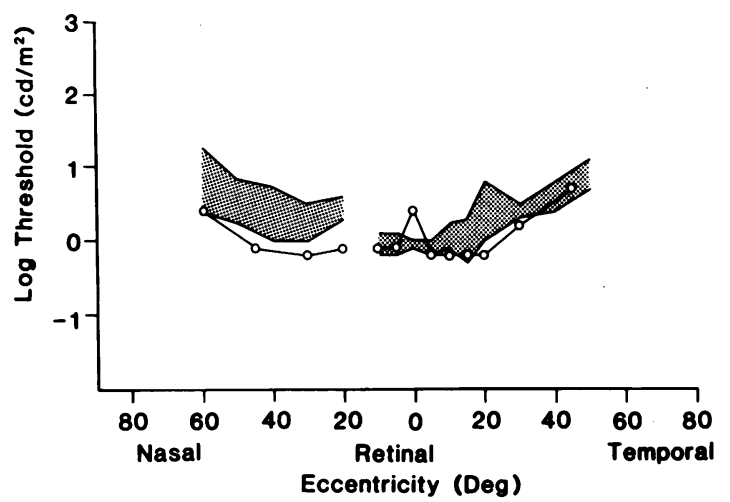

Fig. 9 Light-adapted flicker thresholds across the horizontal meridian of the right eye of patient 2 for a $25 \mathrm{~Hz}$ white test stimulus (open circles). Shaded region represents the range of flicker thresholds for 4 normal observers.

fovea. In contrast to patient 1 this patient has a dystrophy that is localised in the macula and affects primarily cone photoreceptors.

The patient's ERG was tested on the initial visit and then was retested 4 years later, with the findings unchanged. The most recent results are shown in Fig. 10. The photopic a- and b-waves (top tracing) are within normal limits, both in amplitude and in implicit time, consistent with the localised nature of the dystrophy. Under dark-adapted conditions (next 3 tracings), the a-wave amplitude and implicit time are normal, as is the b-wave implicit time, but the b-wave amplitude is supernormal, even with the lowintensity blue flash (Table 1). The b-wave amplitudeintensity function (Fig. 11) confirms these findings. At low flash intensities the $b$-wave is somewhat larger than normal and becomes considerably larger than normal with higher flash intensities.

\section{CASE 3: CONE DYSTROPHY}

A 31-year-old white woman's chief complaint was poor central acuity, which she had experienced since 5 years of age. The patient did not complain of photosensitivity, poor colour vision, or nyctalopia, and specifically mentioned that her acuity was better at night. The patient was otherwise in good health and was not taking any medication. A review of the family pedigree was noninformative, since the patient was adopted.

$$
\begin{aligned}
& \text { CONE DYSTROPHY } \\
& 100 \mu \mathrm{V} \frac{L_{20}}{\mathrm{~ms}}
\end{aligned}
$$

30 Y/O WF

Fig. 10 ERG responses for the right eye of patient 2 (left column) and for a typical normal observer (right column) under the conditions indicated. Vertical bars represent $90 \%$ confidence limits for the mean of the normal responses. Calibration bars are shown at the top.

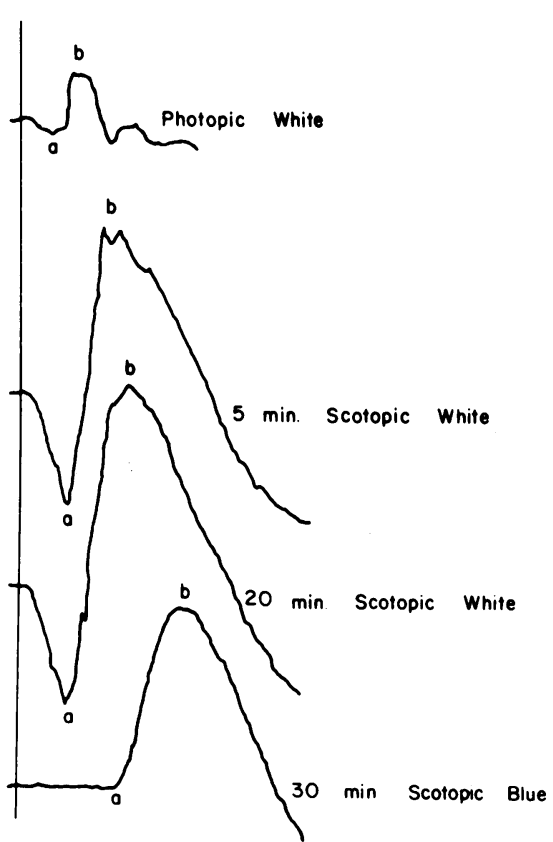

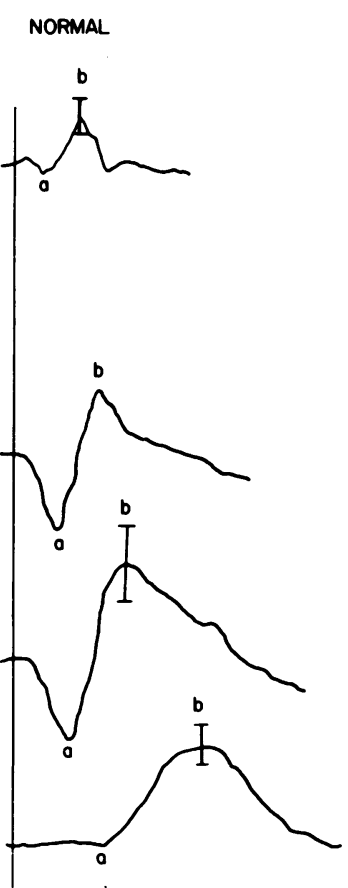




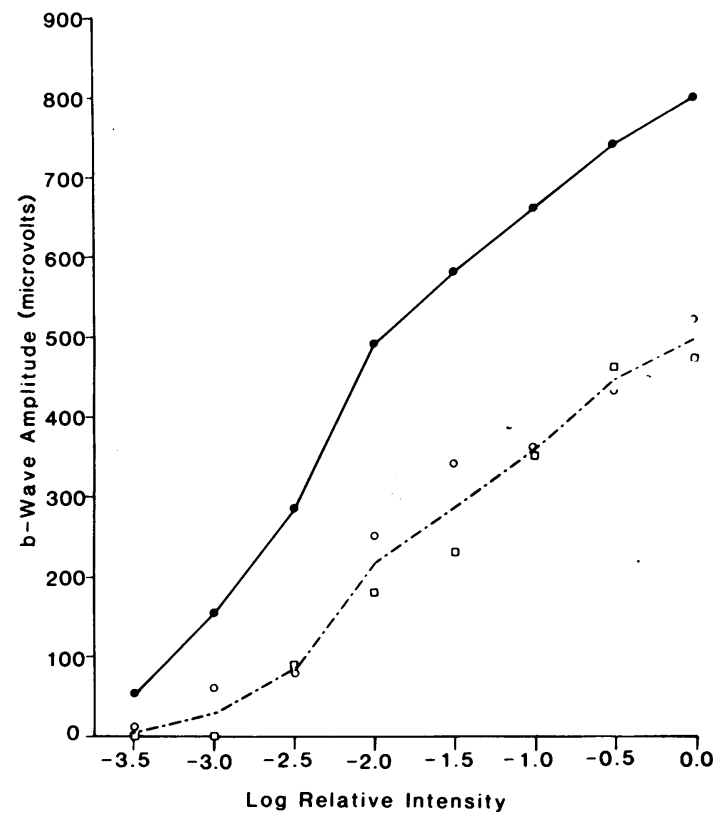

Fig. $11 A$-wave amplitude-intensity function for the right eye of patient 2 (filled circles) and for 2 normal observers (open symbols). Dashed line connects the mean of the 2 normal values.

Visual acuity was correctable to $10 / 60+1$ OD with a $+4 \cdot 75+\cdot 50 \times 100$, and $10 / 50+1$ OS with a $+5 \cdot 25+\cdot 25 \times 70$ using a Feinbloom chart. The patient read J-7 at 12 inches $(30 \mathrm{~cm})$. External examination showed no abnormalities of ocular motility. Slit-lamp

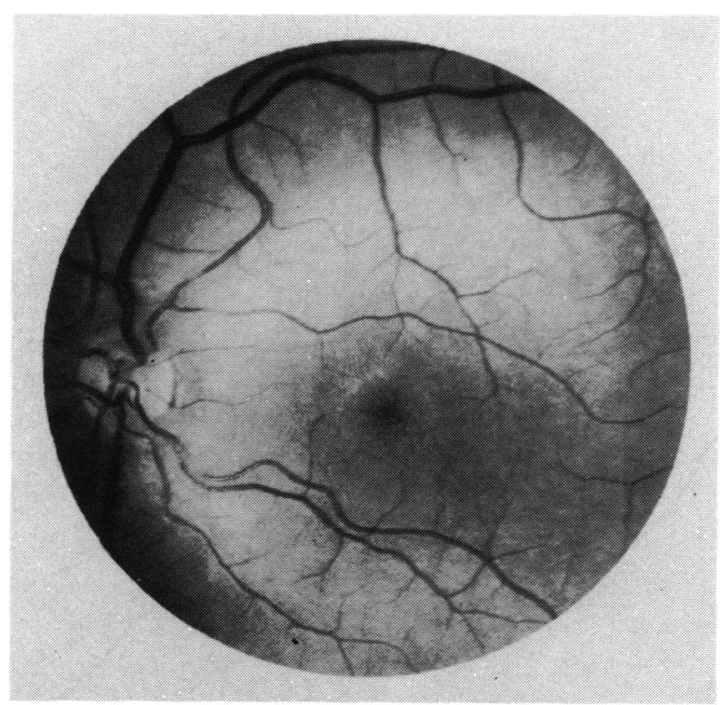

Fig. 12 Fundus photograph of the left eye of patient 3 , depicting an atrophic macular lesion.

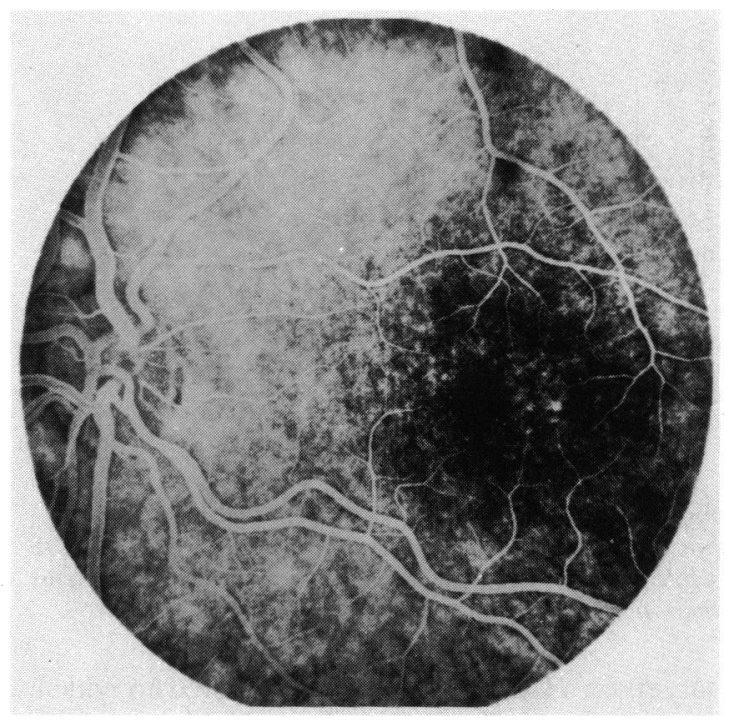

Fig. 13 Fluorescein angiogram of the left eye of patient 3, showing window defects within the macula.

examination of the cornea, anterior chamber, lens, and vitreous was normal. Fundus examination showed normal optic discs and retinal vessels. There was no evidence of any peripheral pigmentary abnormalities. The foveas exhibited bilateral, somewhat subtle atrophic changes that had the appearance of a bull's-eye pattern (Fig. 12). Fluorescein angiography showed bilateral window defects within the fovea, which somewhat highlighted the bull's-eye appearance of her atrophic changes (Fig. 13).

Visual field testing with the 4-e-II and 2-e-II test

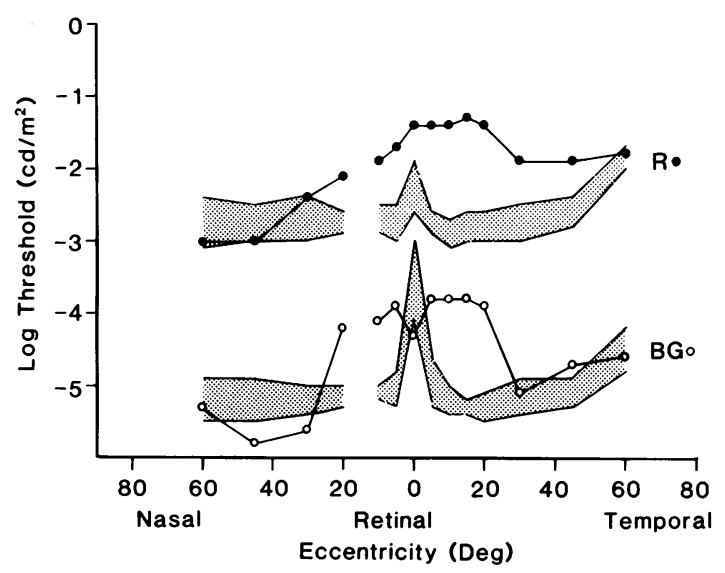

Fig. 14 Absolute thresholds across the horizontal meridian of the right eye of patient 3 for long wavelength (filled circles) and middle wavelength (open circles) test stimuli. Shaded regions represent the range of thresholds for 5 normal observers. 


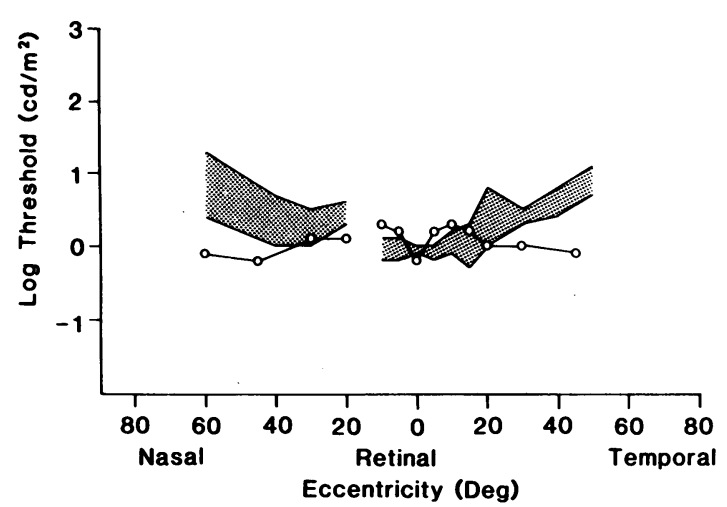

Fig. 15 Light-adapted flicker thresholds across the horizontal meridian of the right eye of patient 3 for a $25 . \mathrm{Hz}$ white test stimulus (open circles). Shaded region represents the range of flicker thresholds for 4 normal observers.

targets on a Goldmann perimeter showed no evidence of peripheral depression but did show bilateral central scotomas. Colour vision testing with the FM 100-hue test demonstrated a bilateral tritan (blue-yellow) defect. The patient was technically unable to perform on the Nagel anomaloscope.
Absolute thresholds across the horizontal meridian are rod-mediated (Fig. 14). As with patient 1, the profile missed the anatomical fovea due to a nonfoveal fixation pattern. Within the central $40^{\circ}$ absolute thresholds were elevated compared with normal, but returned to normal values at the greater eccentricities. Cone flicker thresholds are presented in Fig. 15. There was a slight loss of cone sensitivity in regions near the fovea but normal cone sensitivity at greater eccentricities. As with the previous patient, the dystrophy was confined to the macular region.

The ERG pattern for this patient has been unchanged for 7 years. The most recent results are shown in Fig. 16. The photopic ERG shows amplitudes and implicit times that are within normal limits, consistent with the localised nature of the dystrophy. The dark-adapted a-wave amplitudes and implicit times, as well as the b-wave implicit times, are normal (Table 1). The b-wave response to the dim blue flash is within normal limits. However, the b-wave elicited by the white flash is of supernormal amplitude. An amplitude-intensity function (Fig. 17) shows that the dark-adapted b-wave is normal at low flash intensities, but becomes supernormal with bright flashes.

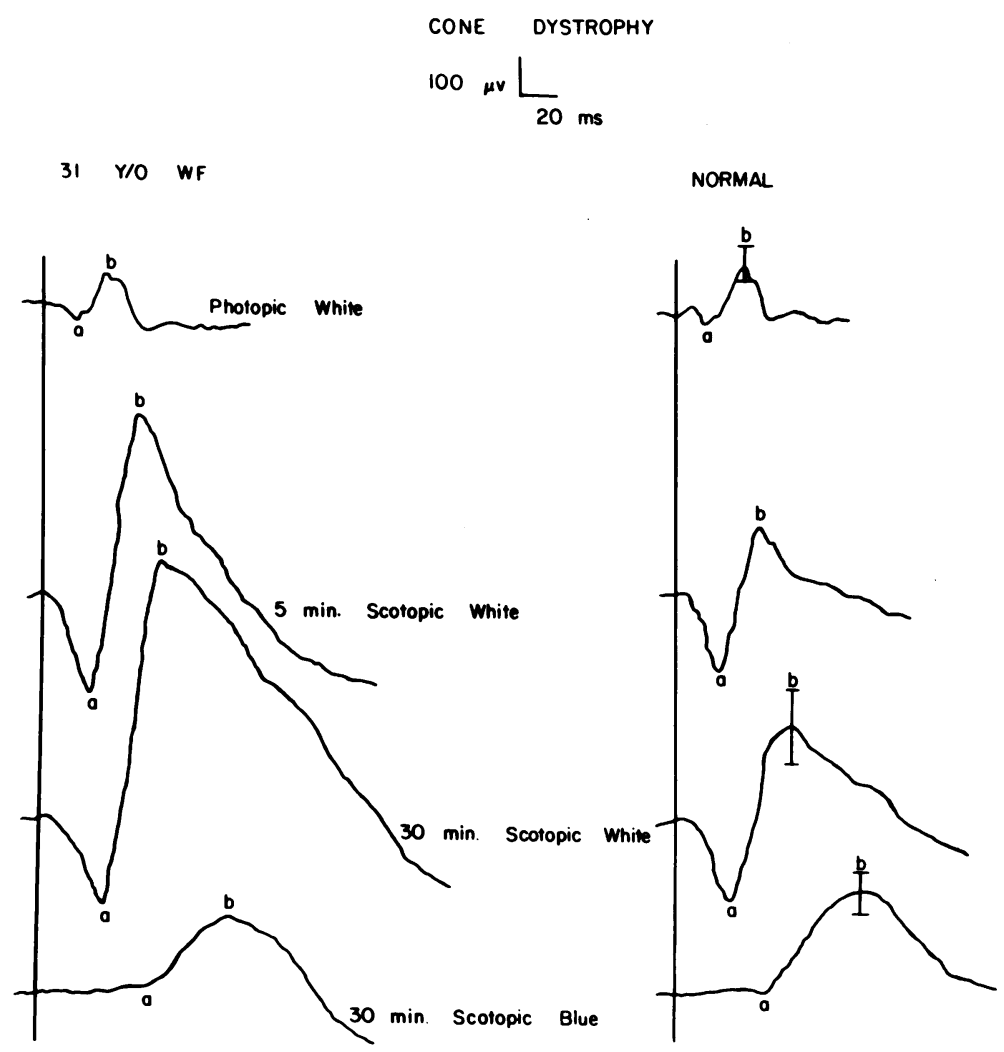

Fig. 16 ERG responses for the right eye of patient 3 (left column) and for a typical normal observer (right column) under the conditions indicated. Vertical bars represent $90 \%$ confidence limits for the mean of the normal responses. Calibration bars are shown at the top. 


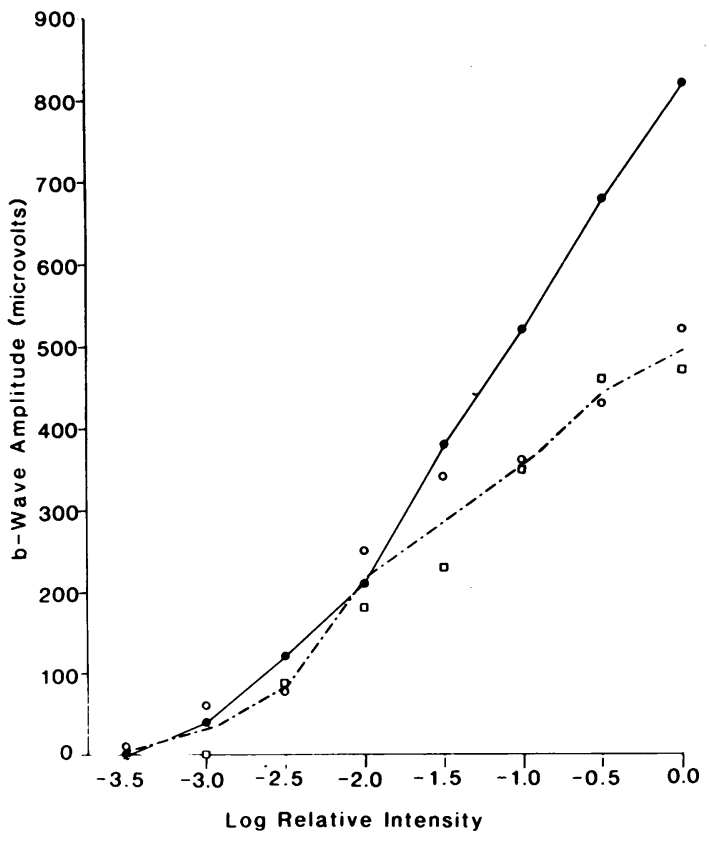

Fig. $17 A$-wave amplitude-intensity function for the right eye of patient 3 (filled circles) and for 2 normal observers (open symbols). Dashed line connects the mean of the 2 normal values.

\section{Discussion}

The 3 patients with cone dystrophy described in this report have a supernormal dark-adapted b-wave response to a moderately intense light flash. A consideration of inheritance patterns suggests that the dystrophy may be an autosomal recessive trait. In several respects patient 1 resembles the 2 patients reported by Gouras et al. ${ }^{10}$ Patient 1 has reduced acuity, decreased colour vision, nyctalopia, a diminished photopic ERG, and a dark-adapted bwave amplitude that is subnormal with dim flashes and supernormal with brighter flashes. Unlike the 2 patients reported previously ${ }^{10}$ patient 1 has a bull'seye macular lesion (rather than a nonspecific macular granularity), with an absence of sensitivity to light. Patients 2 and 3 also have a supernormal darkadapted b-wave, but they appear to represent a different type, or perhaps stage, of cone dystrophy. For both patients the dystrophy is restricted to the macular region rather than being a diffuse cone-rod loss. The scotopic b-wave is normal or larger than normal at low flash intensities, rather than subnormal as with patient 1 .

The pathogenesis of the supernormal scotopic bwave is uncertain. The ERG b-wave is thought to arise predominantly from the Mueller cells in response to changes in the concentration of extracellular potassium that result from the activity of retinal neurons. ${ }^{14}$ The supernormal scotopic b-wave observed in these patients with cone dystrophy may be due to an abnormality in this process. Alternatively Gouras et al. ${ }^{10}$ have suggested that the abnormal ERG may result from an increase in the level of cyclic guanosine monophosphate (cGMP) in the rod photoreceptors, which in turn leads to an abnormal photoreceptor response.

Other conditions besides cone dystrophy have been reported in association with a supernormal ERG in humans, including albinism, ${ }^{15}$ hyperthyroidism, ${ }^{16}$ corticosteroid administration, ${ }^{17}$ optic atrophy, ${ }^{18}$ and retinal vascular disorders. ${ }^{19} \mathrm{We}$ have not been able to implicate any of these factors in the abnormal ERG response in our patients. A supernormal b-wave has also been reported to accompany an increase in $\mathrm{Po}_{2}$ in the isolated perfused cat eye. ${ }^{20}$ However, the relationship between this finding and the results from our patients is uncertain. Although the pathogenesis of the supernormal scotopic b-wave in these patients with cone dystrophy is unclear, the abnormal b-wave represents a diagnostic device that may prove useful in the clinical delineation of cone dystrophy genocopies, which currently appear to be a heterogeneous group of conditions under the same diagnostic label.

Supported by NIH Training Grant EY07038, NIH Core Grant EY01792, and a grant from the National Retinitis Pigmentosa Foundation.

We thank Dr M. F. Goldberg and Ms M. Gere for helpful comments on the manuscript.

\section{References}

1 Sorsby A, Davey HE. Dominant macular dystrophy. Br J Ophthalmol 1955; 39: 385-97.

2 Goodman G, Ripps H, Siegel IM. Cone dysfunction syndromes. Arch Ophthalmol 1963; 70: 214-31.

3 Berson EL, Gouras P, Gunkel RD. Progressive cone degeneration, dominantly inherited. Arch Ophthalmol 1968; 80: 77-83.

4 Krill AE, Deutman AF. Dominant macular degenerations; the cone dystrophies. Am J Ophthalmol 1972; 73: 352-69.

5 Krill AE, Deutman AF, Fishman M. The cone degenerations. Doc Ophthalmol 1973; 35: 1-80.

6 François J, DeRouck A, Verriest G, et al. Progressive generalized cone dysfunction. Ophthalmologica 1974; 169: 255-84.

7 Berson EL, Gouras P, Gunkel RD. Progressive cone-rod dystrophy. Arch Ophthalmol 1968; 80: 68-76.

8 Hittner HM, Murphree AL, Garcia CA, et al. Dominant conerod dystrophy. Doc Ophthalmol 1975; 39: 29-52.

9 Fishman GA. Progressive human cone-rod dysfunction (dystrophy). Trans Am Acad Ophthalmol Otolaryngol 1976; 81: 716-24.

10 Gouras P, Eggers HM, MacKay CJ. Cone dystrophy, nyctalopia and supernormal rod responses: a new retinal degeneration. Arch Ophthalmol 1983; 101: 718-24.

11 Fishman GA, Buckman G, Van Every T. Fundus flavimaculatus: a clinical classification. Doc Ophthalmol Proc Ser 1977; 13: 213-20.

12 Massof RW, Finkelstein D. Two forms of autosomal dominant primary retinitis pigmentosa. Doc Ophthalmol 1981; 51: 289-346. 
13 King-Smith PE, Carden D. Luminance and opponent-color contributions to visual detection and adaptation and to temporal and spatial integration. J Opt Soc Am 1976; 66: 709-17.

14 Kline R, Ripps H, Dowling JE, Generation of b-wave currents in the skate retina. Proc Natl Acad Sci USA 1978; 75: 5727-31.

15 Krill AE, Lee GB. The electroretinogram in albinos and carriers of the ocular albino trait. Arch Ophthalmol 1963; 69: 32-8.

16 Pearlman JT, Burian HM. Electroretinographic findings in thyroid dysfunction. Am J Ophthalmol 1964; 58: 216-25.

17 Zimmerman TJ, Dawson WW, Fitzergald CR. Electroretino- graphic changes in normal eyes during administration of prednisone. Ann Ophthalmol 1973; 5: 757-65.

18 Feinsod M, Rowe H, Auerbach E. Changes in the electroretinogram in patients with optic nerve lesions. Doc Ophthalmol 1971; 29: $169-200$

19 Henkes HE. Electroretinography. An evaluation of the influence of the retinal and general metabolic condition on the electrical response of the retina. Am J Ophthalmol 1957; 43: 67-81.

20 Niemeyer G. ERG dependence on flow rate in the isolated and perfused mammalian eye. Brain Res 1973; 57: 203-7. 